\title{
Clinical course and outcome of patients with high-level microsatellite instability cancers in a real-life setting: a retrospective analysis
}

This article was published in the following Dove Press journal:

OncoTargets and Therapy

29 March 2017

Number of times this article has been viewed

\author{
Naama Halpern' \\ Yael Goldberg² \\ Luna Kadouri² \\ Morasha Duvdevani² \\ Tamar Hamburger ${ }^{2}$ \\ Tamar Peretz ${ }^{2}$ \\ Ayala Hubert ${ }^{2}$
}

'Institute of Oncology, The Chaim Sheba Medical Center, Tel Hashomer, Israel; ${ }^{2}$ Sharett Institute of Oncology, Hadassah Medical Center, Hebrew University, Jerusalem, Israel
Correspondence: Naama Halpern Institute of Oncology, The Chaim Sheba Medical Center, Tel Hashomer 526560 I, Israel

Tel +97235305338

Fax +97235304958

Email naama.halpern@sheba.health.gov.il
Background: The prognostic and predictive significance of the high-level microsatellite instability (MSI-H) phenotype in various malignancies is unclear. We describe the characteristics, clinical course, and outcomes of patients with MSI-H malignancies treated in a real-life hospital setting.

Patients and methods: A retrospective analysis of MSI-H cancer patient files was conducted. We analyzed the genetic data, clinical characteristics, and oncological treatments, including chemotherapy and surgical interventions.

Results: Clinical data of 73 MSI-H cancer patients were available. Mean age at diagnosis of first malignancy was 52.3 years. Eight patients $(11 \%)$ had more than four malignancies each. Most patients $(76 \%)$ had colorectal cancer (CRC). Seventeen patients $(23 \%)$ had only extracolonic malignancies. Eighteen women (36\%) had gynecological malignancy. Nine women (18\%) had breast cancer. Mean follow-up was 8.5 years. Five-year overall survival and disease-free survival of all MSI-H cancer patients from first malignancy were $86 \%$ and $74.6 \%$, respectively. Five-year overall survival rates of stage 2, 3, and 4 MSI-H CRC patients were $89.5 \%, 58.4 \%$, and $22.9 \%$, respectively.

Conclusion: Although the overall prognosis of MSI-H cancer patients is favorable, this advantage may not be maintained in advanced MSI-H CRC patients.

Keywords: microsatellite instability, malignancy, treatment, outcome

\section{Introduction}

Precision medicine is becoming more significant in the routine clinical practice of the oncologist. High-level microsatellite instability (MSI-H) describes a condition of genetic hypermutability. ${ }^{1,2}$ The MSI-H phenotype can result from methylation of the MLH1 gene promoter, which is usually considered a sporadic event, or from a mutation in one of the mismatch repair (MMR) genes, which is the hallmark of Lynch syndrome (LS). ${ }^{3} \mathrm{LS}$ is the most common cause of hereditary colorectal cancer (CRC) and is characterized by the predisposition to a spectrum of malignancies. ${ }^{4}$

MSI-H can be found in various malignancies, and most of them are LS-associated cancers. ${ }^{5}$ Approximately $15 \%-20 \%$ of CRC will exhibit MSI-H, mainly sporadic. ${ }^{3}$

The importance of identifying MSI-H CRCs as a surrogate for LS diagnosis is unquestionable and is recommended by the NCCN guidelines for all CRC patients below 70 years of age. ${ }^{6}$ Surveillance protocols and preventive measures reduce cancer incidence and mortality in these patients. ${ }^{7,8}$

In the last decade, efforts have been invested to expand the knowledge and understanding of the clinical significance of MSI-H as a biomarker. Since the 
early 1990s, evidence accumulated suggests that MSI-H CRCs have a favorable prognosis. These tumors will less likely involve lymph nodes or distant organs; hence, they are more likely to be diagnosed at an early stage. ${ }^{9-11}$ It is now widely accepted that stage $2 \mathrm{MSI}-\mathrm{H}$ CRC patients have a favorable prognosis. ${ }^{12-14}$ Whether these patients benefit from adjuvant chemotherapy is controversial; some studies suggest they do not benefit, while others conclude that the relative benefit from chemotherapy is similar to that of microsatellite stable (MSS) patients. ${ }^{11-13,15}$

MSI-H is less prevalent in stage 3 and 4 CRC patients and is found only in approximately $10 \%$ and $5 \%$ of patients, respectively. ${ }^{11}$ The impact of MSI-H on the prognosis of these patients is not clear, neither if they benefit differently from chemotherapy, biological, and immunotherapy treatments. ${ }^{16-24}$

The prognostic and predictive value of MSI-H in other malignancies is being studied but has not been established. ${ }^{25-28}$ Fang et $\mathrm{al}^{25}$ found MSI-H tumors in $11 \%$ of patients with gastric cancer undergoing curative surgery. MSI-H patients had a significantly better overall survival (OS) rate compared to that of MSS patients in this study. In contrast, a different study did not find a clear influence of MSI status on OS of gastric cancer patients. ${ }^{26}$ Rouprêt et al described a better prognosis for patients with MSI-H invasive upper urinary tract transitional cell carcinomas. The rate of MSI-H in this study was $20 \% .{ }^{27} \mathrm{MSI}-\mathrm{H}$ status was found as a marker for poor prognosis in early-stage endometrial endometrioid adenocarcinomas. ${ }^{28}$

The hypermutability state of MSI-H tumors makes them preferred candidates for immunotherapies, and evidence for MSI-H serving as a biomarker for patient selection to these treatments is emerging. ${ }^{24}$

Much of the knowledge about the clinical course and outcome of MSI-H cancer patients is derived from data of highly selected patients participating in clinical trials, a setting that does not always truly represent routine daily practice.

The current study was conducted to get a better perspective on the clinical course and outcomes of MSH-H cancer patients in a real-life, hospital-based setting.

\section{Patients and methods}

Medical records of patients who had at least one MSI-H malignancy and were treated or followed up in Hadassah Hebrew University Medical Center from 2004 to 2015 were reviewed. We analyzed clinical, genetic, and pathological characteristics as well as the oncological course, including chemotherapy administrated and surgical interventions. All patients signed informed consent to genetic counseling.
The research plan was reviewed and approved by the institutional Helsinki committee (institutional review board).

Most patients had tumors tested for MSI after genetic counseling due to personal or familial background of malignancies. Some of the patients had evaluation of MSI status for treatment decision (stage 2 CRC) and then referred to genetic counseling based on MSI-H phenotype.

Tumors were considered MSI-H based on polymerase chain reaction (PCR) for MSI and/or if they were determined MMR deficient by immunohistochemistry (IHC). The two tests are accepted by the NCCN guidelines and other publications as interchangeable, measuring the same biological effect. ${ }^{6,16}$ We also considered tumors as MSI-H if they were LS-associated tumors in patients with a germline pathogenic mutation in one of the MMR genes. Although not all tumors from patients with multiple malignancies were tested for MSI, we did describe them in this cohort.

The disease-free survival (DFS), recurrence-free survival (RFS), and OS distributions were estimated using the Kaplan-Meier method. RFS was defined as the time from diagnosis of the malignancy to first documented specific malignancy recurrence. Statistical analyses were carried out using IBM SPSS version 19.

\section{Compliance with ethical standards}

All patients signed informed consent to genetic counseling. Research plan was reviewed and approved by the Ethics (Helsinki) Committee at Hadassah University Hospital. The ethics committee did not require patients' informed consent for this study, as this is a retrospective, anonymous review of medical records.

\section{Results}

\section{Entire cohort}

Clinical data of 73 MSI-H cancer patients were analyzed (Table 1). Forty-nine patients (67\%) were females. Mean age at diagnosis of first malignancy was 52.3 years. Germline pathogenic mutation in one of the MMR genes was identified in half of the patients. Most patients (76\%) had CRC, and $23 \%$ had only extracolonic malignancies. Eight patients (11\%) had more than four malignancies each.

Mean follow-up for all patients was 8.5 years (range 0.3-49.5). Five-year OS and DFS rates of all MSI-H cancer patients from first malignancy diagnosis were $86 \%$ and $74.6 \%$, respectively.

\section{CRC cohort}

Fifty-six patients had $70 \mathrm{CRCs}$; six patients (11\%) had synchronous tumors, one of them presented with five primaries. 
Table I Characteristics of all MSI-H patients

\begin{tabular}{lll}
\hline Characteristic & $\begin{array}{l}\text { All MSI-H patients } \\
(\mathbf{N}=\mathbf{7 3})\end{array}$ & $\%$ \\
\hline Mean age at first disease (years) & $52.3(27-9 I)$ & \\
First diagnosis 550 years & 33 & 54.8 \\
Female gender & 49 & 67.1 \\
Germline pathogenic MMR mutation & 36 & 49.3 \\
Missing proteins (IHC) & & \\
MLHI/PMS2 & 37 & 50.6 \\
MSH2/MSH6 & 18 & 24.6 \\
Missing data & 18 & 24.6 \\
Number of malignancies per patient & & \\
I & 48 & 65.7 \\
2 & 12 & 16.4 \\
3 & 5 & 6.8 \\
>4 & 8 & 10.9 \\
Patients with & & 76.7 \\
Colorectal cancer & 56 & 36.7 \\
GY cancer (all women =49) & 18 & 45 \\
GY cancer (all women no & 18 & \\
prophylactic surgery =40) & & 5.5 \\
GU (excluding prostate) & 4 & 18.3 \\
Breast (all women) & 9 & 9.5 \\
Skin (all) & 7 & 0 \\
Other & 9 & 23.2 \\
Brain & 0 & \\
Extracolonic malignancy only & 17 & \\
\hline
\end{tabular}

Note: Data presented as mean (range) or $\mathrm{n}$.

Abbreviations: MSI-H, high-level microsatellite instability; MMR, mismatch repair; IHC, immunohistochemistry; GY, gynecological; GU, genitourinary.

Four patients had metachronous (7\%) tumors (Table 2). As expected, most CRCs were located in the right colon (58\%) and diagnosed at an early stage (67\% at stages 1 and 2 ). Eighteen percent of the tumors were poorly differentiated.

Twenty-five percent of the tumors were tested for BRAF V600E mutation, and they were all wild type (WT). Five-year survival of all CRC patients was $83.6 \%$. Five-year RFS of all CRC patients was $74.8 \%$.

\section{Stage 2 CRC}

Not surprisingly, stage $2 \mathrm{CRC}$ was the most common malignancy in our cohort (Table 2). Two-thirds of stage 2 MSI-H CRC patients did not receive adjuvant chemotherapy. When given, adjuvant chemotherapy was based on fluoropyrimidines. One stage 2 patient received adjuvant treatment, including oxaliplatin.

Five-year OS and RFS rates of stage 2 MSI-H CRC patients were good as expected, $89.5 \%$ and $89.7 \%$, respectively.

\section{Stage 3 CRC}

Eleven patients had stage 3 MSI-H CRC as first CRC (Table 2). Most of them (72\%) received adjuvant oxaliplatin-based chemotherapy. Recurrent metastatic disease was diagnosed in over half of our stage 3 MSI-H CRC patients, with 5-year
OS and RFS rates of $58.4 \%$ and $44.7 \%$, respectively. Most of stage 3 patients with recurrent disease had high-risk features for recurrence (T4 tumor, perforation, extensive lymph node involvement, and perineural invasion). Four out of these six patients received adjuvant chemotherapy with an oxaliplatinbased regimen. Mean time for the diagnosis of recurrent metastatic disease was 17.5 months (range: 7-48 months).

\section{Metastatic CRC}

Fifteen patients had MSI-H metastatic CRC (Table 2). Seven of them had metastatic disease at first presentation of CRC.

Forty percent of these patients had a germline pathogenic MMR gene mutation identified. BRAF V600E status was known for six patients (40\%); all of them were WT. Five of the patients with no information regarding BRAF V600E status had a pathogenic MMR gene mutation and so are unlikely to be BRAF mutated. Three patients had MLH1 deficiency on IHC and no information about BRAF V600E status. KRAS status was known in $60 \%$ of the metastatic tumors, with a mutation detected in two $(13 \%)$ of them.

Only $20 \%$ of patients had liver metastases as first site of metastatic disease. Metastasectomy rate was relatively high with eight patients (53\%) undergoing surgery (one hepatectomy, two cytoreductions [CRSs], one pelvic exenteration, two lymph-node dissections, one skeletal metastasis resected, and one oophorectomy). Five-year OS for metastatic MSI-H CRC was $22.9 \%$. Median OS was 18.4 months.

Treatments administrated to metastatic MSI-H CRC patients were heterogeneous, and we did not recognize any trend toward prolonged responses to a specific regimen.

Two patients have no evidence of disease for over 5 years; one had CRS and hyperthermic intraoperative peritoneal chemotherapy (HIPEC) surgery and did not receive any systemic treatment for metastatic disease. She has a germline pathogenic mutation in MSH6. The second patient had retroperitoneal lymph node dissection and 6 months of FOLFOX bevacizumab treatment. She has MLH1 PMS2 deficiency on IHC, her BRAF status is not known, and she did not complete germline mutation analysis.

Two additional patients had a prolonged course, both of them had metastasectomy; one underwent CRS and HIPEC and received FOLFIRI-bevacizumab for a total of 14 months in a few intervals. He is alive with disease 42 months from initial diagnosis. IHC showed MLH1 deficiency, and BRAF is WT. A second patient had a pathogenic mutation in MSH6. He underwent retroperitoneal lymph node dissection and received various chemotherapy lines. He succumbed to cancer 48 months after the diagnosis of metastatic disease. 
Table 2 Characteristics of all MSI-H CRC patients

\begin{tabular}{|c|c|c|c|c|c|}
\hline Characteristic & $\begin{array}{l}\text { All CRC patients } \\
(\mathrm{N}=56, \text { tumors }=70)^{\mathrm{a}}\end{array}$ & $\begin{array}{l}\text { Stage I } \\
(\mathrm{N}=3)^{\mathrm{b}}\end{array}$ & $\begin{array}{l}\text { Stage } 2 \\
(\mathrm{~N}=35)^{\mathrm{b}}\end{array}$ & $\begin{array}{l}\text { Stage } 3 \\
\left(\mathrm{~N}=I^{\prime}\right)^{\mathrm{b}}\end{array}$ & $\begin{array}{l}\text { Stage } 4 \\
(N=15)^{c}\end{array}$ \\
\hline Mean age at first CRC diagnosis (years) & $53.4(27-9 \mid)$ & $50(47-53)$ & $51.8(27-86)$ & $63.7(47-9 \mid)$ & $52.3(27-91)$ \\
\hline Diagnosis of first $C R C \leq 50$ years & $24(43 \%)$ & $2(67 \%)$ & $16(46 \%)$ & $2(18 \%)$ & $8(53 \%)$ \\
\hline Germline pathogenic MMR mutation & $25(45 \%)$ & $2(67 \%)$ & 17 (49\%) & $3(27 \%)$ & $6(40 \%)$ \\
\hline \multicolumn{6}{|l|}{ Tumor site $(\mathrm{N}=70)$} \\
\hline Right & $40(57 \%)$ & $2(67 \%)$ & $26(74 \%)$ & $4(36 \%)$ & $9(60 \%)$ \\
\hline Transverse & $6(9 \%)$ & 0 & $5(14 \%)$ & 0 & 0 \\
\hline Left & $13(19 \%)$ & I (33\%) & $3(9 \%)$ & $4(36 \%)$ & $5(33 \%)$ \\
\hline Rectum & $4(6 \%)$ & 0 & $\mathrm{I}(3 \%)$ & $2(18 \%)$ & $\mathrm{I}(7 \%)$ \\
\hline Missing data & $7(10 \%)$ & 0 & 0 & I (9\%) & 0 \\
\hline \multicolumn{6}{|l|}{ Tumor differentiation $(\mathrm{N}=70)$} \\
\hline Well moderate & $42(60 \%)$ & I (33\%) & $28(80 \%)$ & $6(54.5 \%)$ & $6(40 \%)$ \\
\hline Poor & $13(19 \%)$ & 0 & $2(6 \%)$ & $5(45.5 \%)$ & $8(53 \%)$ \\
\hline Missing data & $15(21 \%)$ & $2(67 \%)$ & $5(14 \%)$ & 0 & I (7\%) \\
\hline Recurrent metastatic disease & NA & 0 & $2(6 \%)$ & $6(54.5 \%)$ & NA \\
\hline Patients with synchronous tumors & $6(11 \%)$ & 0 & $4(11 \%)$ & I (9\%) & $\mathrm{I}(7 \%)$ \\
\hline Patients with metachronous tumors & $4(7 \%)$ & 0 & $3(9 \%)$ & I (9\%) & 0 \\
\hline \multicolumn{6}{|l|}{ First metastatic site } \\
\hline Liver & NA & NA & NA & NA & $3(20 \%)$ \\
\hline Lung & NA & NA & NA & NA & I (7\%) \\
\hline Retroperitoneum (including LN) & NA & NA & NA & NA & $4(27 \%)$ \\
\hline Peritoneum & NA & NA & NA & NA & $3(20 \%)$ \\
\hline Bones & NA & NA & NA & NA & I (7\%) \\
\hline Ovary & NA & NA & NA & NA & I (7\%) \\
\hline Other/multiple sites & NA & NA & NA & NA & $2(13 \%)$ \\
\hline Metastasectomy & NA & NA & NA & NA & $8(53 \%)$ \\
\hline
\end{tabular}

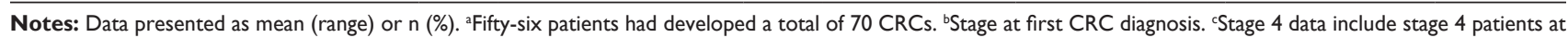
diagnosis and recurrence.

Abbreviations: MSI-H, high-level microsatellite instability; CRC, colorectal cancer; MMR, mismatch repair; NA, not applicable; LN, lymph nodes.

\section{Extracolonic malignancies cohort Gynecological (GY) malignancies}

Eighteen women had a GY malignancy (Table 3). There were 49 women in this cohort; 9 of them (18\%) had prophylactic hysterectomy and oophorectomy before they had a GY cancer. This makes a rate of $45 \%$ GY malignancy in women who did not have a prophylactic surgery.

Mean age at diagnosis of GY malignancy was 52.8 (40-86) years, and $33 \%$ of patients were diagnosed with GY malignancy at or before age 50. As expected, most GY malignancies were uterine cancers, half of them were endometrioid type. There were two cases of ovarian cancer (serous and clear cell histology) and one case of uterine cervix cancer with endometrioid histology. Most GY cancers were diagnosed at an early stage ( $78 \%$ ), and only $27 \%$ of patients got adjuvant chemotherapy. Five-year OS rate for GY MSI-H malignancy was $100 \%$, and 5-year RFS rate was $93.3 \%$.

One patient had recurrent metastatic disease; she was 43 years when diagnosed with stage 1 endometrial cancer,
Table 3 Patients with gynecological malignancies

\begin{tabular}{lll}
\hline Characteristic & $\begin{array}{l}\text { Number } \\
\text { of patients } \\
(\mathbf{N}=\mathbf{1 8})\end{array}$ & $\%$ \\
\hline Mean age at diagnosis (years) & $52.8(40-86)$ & \\
Diagnosis 5 50 years & 6 & 33.3 \\
Pathogenic germline MMR mutation & 12 & 66.7 \\
Tumor site & & \\
$\quad$ Uterine & 16 & 84.2 \\
Ovary & 2 & 10.5 \\
Cervix & 1 & 5.3 \\
Stage at diagnosis & & \\
I & 15 & 78.9 \\
2 & 0 & 0 \\
3 & $\mathrm{I}$ & 5.3 \\
4 & 0 & 0 \\
Missing data & 3 & 15.8 \\
Adjuvant chemotherapy & 5 & 27.8 \\
Adjuvant radiotherapy & 7 & 38.8 \\
Recurrent metastatic disease & $\mathrm{I}$ & 5.5 \\
\hline
\end{tabular}

Notes: Data presented as mean (range) or $n$. ${ }^{2}$ One patient had synchronous ovarian and uterine tumors.

Abbreviation: MMR, mismatch repair. 
mixed serous, and endometrial histology. The patient underwent surgery and completed adjuvant chemotherapy with carboplatin and paclitaxel but presented with metastatic disease 7 months after initial diagnosis. A pathogenic mutation in MSH6 was detected. She is currently alive with disease, $>3$ years after diagnosis of metastatic disease, with a partial response to anti-PD-1 therapy.

\section{Breast cancers}

Nine women (18\%) had 11 breast cancers (Table 4). Most breast cancers $(78 \%)$ were invasive ductal carcinomas, $55 \%$ were estrogen receptor positive, none were human epidermal growth factor receptor 2 positive, and 22\% were triple negative. Forty-four percent of breast cancers were diagnosed at stage 3 , and two women had bilateral breast cancers. None of the women had recurrent metastatic breast cancer.

\section{Other extracolonic malignancies}

Seventeen patients (23\%) had only extracolonic cancers. Four patients $(5 \%)$ had six genitourinary malignancies (excluding prostate cancer). Three patients had prostate cancer. Seven patients $(9 \%)$ had skin cancers (two malignant melanomas, three basal cell carcinomas, one squamous cell carcinoma, and one sebaceous carcinoma). Other malignancies were MALT lymphoma of the stomach, lung cancer, colon

Table 4 Patients with breast cancers

\begin{tabular}{|c|c|c|}
\hline Characteristic & $\begin{array}{l}\text { Number of } \\
\text { patients } \\
(\mathbf{N}=9)\end{array}$ & $\%$ \\
\hline Mean age at diagnosis (years) & $61.7(43-78)$ & \\
\hline Diagnosis $\leq 50$ years & 2 & 22.2 \\
\hline Pathogenic germline MMR mutation & 5 & 55.5 \\
\hline \multicolumn{3}{|l|}{ Stage at diagnosis } \\
\hline I & 4 & 44.5 \\
\hline 2 & 1 & 11 \\
\hline 3 & 4 & 44.5 \\
\hline 4 & 0 & 0 \\
\hline \multicolumn{3}{|l|}{ Histology } \\
\hline Invasive ductal carcinoma & 7 & 77.8 \\
\hline Invasive lobular carcinoma & 1 & II \\
\hline Papillary carcinoma & 1 & $\mathrm{II}$ \\
\hline \multicolumn{3}{|l|}{ Receptors } \\
\hline ER positive & 5 & 55.5 \\
\hline HER2 positive & 0 & 0 \\
\hline Triple negative & 2 & 22.2 \\
\hline Bilateral breast cancer & 2 & 22.2 \\
\hline Recurrent metastatic disease & 0 & 0 \\
\hline
\end{tabular}

Note: Data presented as mean (range) or $\mathrm{n}$.

Abbreviations: MMR, mismatch repair; ER, estrogen receptor; HER2, human epidermal growth factor receptor 2 . carcinoid, and two cases of thyroid cancer (medullary and papillary histology).

Only one patient had gastric cancer. She has a pathogenic mutation in MSH6. She was diagnosed with metastatic disease to her adnexa at the age of 77 . She had partial gastrectomy and oophorectomy. She received only four cycles of chemotherapy with carboplatin and fluorouracil with low tolerance. Since then she is receiving an allogeneic vaccine and is alive 4 years after diagnosis. Lately, she had a solitary lung nodule that is highly suspicious for metastatic disease, continuing surveillance.

\section{Discussion}

In this study, we summarize the molecular and clinical data of MSI-H cancer patients. This enables a view of real-life practice and outcomes of this unique patient population.

A noticeable finding in our cohort is the fact that $67 \%$ of patients were females. One can argue that this is solely due to a referral bias (more women referred to genetic counseling due to endometrial cancer), but an association between female gender and MSI-H has been described previously in the literature. ${ }^{10,15,29,30}$ Most of these published data are based on routine screening of CRC samples, thus, the possibility of a referral bias is less likely. Overrepresentation of the female gender is established in the subset of sporadic, MSI-H, BRAF-mutated CRC, and might be explained by the effects of estrogen withdrawal with increasing age, as estrogen protects against tumor instability by decreasing the promoter methylation. ${ }^{31-34}$ BRAF status was known only for nine women in our cohort, all of them were WT.

The outcomes of our MSI-H cancer patients cohort confirm the previously described favorable prognosis of MSI-H CRC and other malignancies exhibiting MSI-H. ${ }^{9-11,25,28,35}$

Our CRC patients group had a 5-year OS rate of $75.9 \%$. This result is much better than the 64.9\% 5-year OS reported by the Surveillance, Epidemiology, and End Results Program (SEER) database from 2005 to $2011 . .^{36,37}$ An important fact that has to be considered when comparing these outcome data is the high percentage of early-stage cases in our cohort; almost $70 \%$ of CRC patients in our cohort were diagnosed with localized (stages 1 and 2) disease, compared to $<40 \%$ in the SEER database.

Looking at per stage survival data, our 5-year OS rates of stage 2 CRC patients were similar to the SEER database localized CRC (stages 1 and 2) 5-year OS rates; 89.5\% vs $90.1 \%$, respectively. ${ }^{38}$ Taking into consideration the fact that the SEER data analysis includes stage $1 \mathrm{CRC}$, our data 
of stage 2 only patients are slightly better. These data are consistent with previous reports of favorable early MSI-H CRC patient prognosis. ${ }^{12,39}$ Hutchins et al ${ }^{15}$ analyzed MSI, KRAS, and BRAF status of almost 2,000 stage 2 and 3 CRC patients randomly assigned between fluorouracil and folinic acid chemotherapy and no chemotherapy in the Quick and Simple and Reliable (QUASAR) trial. Most patients were stage 2. They found that the recurrence rate for MSI-H tumors was half that of MMR-proficient tumors (11\% vs $26 \%$ ) and was not significantly different between BRAF mutant and WT tumors. They found no evidence that patients with MSI-H fail to respond to chemotherapy and concluded that the absolute benefit from chemotherapy is likely to be proportional to the absolute risk of recurrence.

We were interested in understanding the impact of the MSI-H phenotype on advanced stage CRC prognosis. Limited data about stage 3 MSI-H CRC patients are available since many publications report the outcomes of stage 2 and 3 patients together. ${ }^{40}$

The few publications from recent years that did stratify patients by stage conclude that the prognosis of stage $3 \mathrm{MSI}-\mathrm{H}$ CRC patients was similar or better compared to MSS CRC patients. ${ }^{21,41}$ Sinicrope et $\mathrm{al}^{22}$ analyzed molecular markers in stage 3 CRC patients treated with FOLFOX \pm cetuximab and found a similar DFS rate of $\sim 70 \%$ for patients with MMR-deficient sporadic or familial subtypes and patients with MMRproficient tumors without BRAF or KRAS mutations.

Our stage 3 MSI-H CRC patients were worrisome with a 5 -year RFS rate of $44.7 \%$. This high recurrence rate is worse than expected and is not explained by overrepresentation of BRAF-mutated or rectal tumors that are considered more aggressive. As described previously, the majority of patients with recurrent stage 3 disease had adverse prognostic factors at diagnosis. Extensive lymph node involvement increased the risk of recurrence in MSI-H tumors with a 5-year DFS rate of $48 \%-57 \% .{ }^{33}$ It is worth noting that the high recurrence rate did not compromise the 5-year OS rate of this group, which was $70 \%$, similar to the reported survival rates by the SEER database for all stage 3 CRCs. ${ }^{37}$

Metastatic MSI-H CRC patients are rare, thus less is known about the significance of MSI-H as a prognostic and predictive marker in this patient group. Our cohort included 15 metastatic MSI-H CRCs. The 5-year OS rate of this group was $22.9 \%$, which is better than the $13 \% 5$-year OS rates reported by the SEER database for stage 4 CRC patients. ${ }^{37}$ Median OS in our MSI-H CRC group was 18.4 months, also slightly better than the median OS of 14 months reported for all metastatic CRC patients. ${ }^{38}$ Median OS for metastatic
MSI-H CRC was 15.4 months in a cohort published by Goldstein et al. ${ }^{16}$ They concluded that compared with historical controls, patients with MSI-H metastatic CRC do not appear to have improved outcomes and they did not find support for MSI-H status predicting differential chemotherapy benefit in metastatic patients. In their cohort, 23\% of patients underwent metastasectomy, with an improved median survival rate of 33 months. Slightly more than half of our metastatic CRC patients had surgery for metastatic disease (excluding primary tumor resection), and only one of those was hepatectomy. Although our numbers were too small to assess the influence of this parameter on patient survival, it is worth noticing that all patients with very long survival underwent metastasectomy.

It is also worth mentioning that a relatively high percent of our patients had peritoneal or retroperitoneal spread as first metastatic site. These data imply that MSI-H CRCs might have a different spreading pattern.

Immunotherapy strategies in oncology play a central role in many different malignancies. MSI-H is now considered a predictive biomarker for a good response to checkpoint inhibitors in CRCs and other malignancies. ${ }^{24}$ The assumed mechanism behind this is the hypermutability state of these tumors that have the potential to encode "nonself" immunogenic antigens. ${ }^{42}$

The prognostic and predictive significance of MSI-H in extracolonic malignancies has been less studied. Unfortunately, the number of extracolonic malignancies in our cohort was too small to enable statistical analysis. This may represent underdiagnosis of MSI-H in patients with extracolonic malignancies. We do describe an unusual prolonged course of a patient with metastatic MSI-H gastric cancer who had no evidence of disease for 4 years with hardly any systemic treatment but a vaccine. Whether the combination of metastasectomy and immunotherapy confers a potential for long-term responses in this unique subset of patients is an interesting question.

Nine women (18\%) had 11 breast cancers in our cohort. There is a debate in the literature whether LS carriers are at increased risk of breast cancer. ${ }^{43-46}$ Almost half of our breast cancer patients were diagnosed with advanced stage cancers, so it is less likely that the reason for this number is overscreening. None of the patients had recurrent metastatic disease. We did not recognize any unique profile of these breast cancers. Our data highlight the need for further research of the risk of breast cancer in LS carriers.

In the majority of our cases, the diagnosis of MSI-H was made after the patients were treated for their malignancy. 
Whether knowing MSI status at initial diagnosis, prior to treatment decisions, will affect treatment management is another interesting issue.

Our study has several limitations. It is retrospective, and the sample size of patients is small. The study was conducted over a decade; MSI tests were not homogenous, as they were performed over the years in different laboratories; treatment standards have changed over time; thus patients might have been managed differently for similar disease. Moreover, choice of therapy for each patient was dependent upon the treating physician. This led to a high variability of treatment protocols and did not allow for sufficient numbers for analysis. Data were collected and analyzed before immunotherapy agents were available for MSI-H cancer patients. Our patient cohort consists mainly of patients referred to genetic counseling, a fact that might lead to underrepresentation of patients with sporadic MSI-H cancers. We did not have BRAF V600E mutation analysis for all patients. Some of them had a pathogenic mutation in one of the MMR genes so are less likely BRAF mutated. Overall, we did not have BRAF V600E status or a pathogenic mutation in MMR gene in 26 patients $(35 \%)$.

Despite its limitations, our study presents a unique combination of genetic and clinical data, including treatments administrated to patients, surgical interventions, and most importantly, patient outcomes. This gives a perspective of real-life practice, challenges, and outcomes of MSI-H cancer patients.

\section{Conclusion}

While confirming the overall good prognosis of the patient population with MSI-H cancers, our study highlights the open question of whether this favorable prognosis is maintained in advanced stages. Our stage 3 MSI-H CRC patients had an unfavorable prognosis with $>50 \%$ recurrence rate. Patients with metastatic MSI-H CRC did not seem to have a favorable prognosis and might have a unique spreading pattern. These data strengthen the need for further research and new, tailored treatment strategies for MSI-H cancer patients.

\section{Disclosure}

The authors report no conflicts of interest in this work.

\section{References}

1. Thibodeau SN, Bren G, Schaid D. Microsatellite instability in cancer of the proximal colon. Science. 1993;260(5109):816-819.

2. Network CGA. Comprehensive molecular characterization of human colon and rectal cancer. Nature. 2012;487(7407):330-337.
3. Cunningham JM, Christensen ER, Tester DJ, et al. Hypermethylation of the hMLH1 promoter in colon cancer with microsatellite instability. Cancer Res. 1998;58(15):3455-3460.

4. Lynch HT, Snyder CL, Shaw TG, Heinen CD, Hitchins MP. Milestones of Lynch syndrome: 1895-2015. Nat Rev Cancer. 2015;15(3): 181-194.

5. Vasen HF, Watson P, Mecklin JP, Lynch HT. New clinical criteria for hereditary nonpolyposis colorectal cancer (HNPCC, Lynch syndrome) proposed by the International Collaborative group on HNPCC. Gastroenterology. 1999;116(6):1453-1456.

6. Hampel H. NCCN increases the emphasis on genetic/familial highrisk assessment in colorectal cancer. J Natl Compr Canc Netw. 2014; 12(5 suppl):829-831.

7. Burn J, Gerdes AM, Macrae F, et al. Long-term effect of aspirin on cancer risk in carriers of hereditary colorectal cancer: an analysis from the CAPP2 randomised controlled trial. Lancet. 2011;378(9809):2081-2087.

8. Lynch HT, Drescher K, Knezetic J, Lanspa S. Genetics, biomarkers, hereditary cancer syndrome diagnosis, heterogeneity and treatment: a review. Curr Treat Options Oncol. 2014;15(3):429-442.

9. Gryfe R, Kim H, Hsieh ET, et al. Tumor microsatellite instability and clinical outcome in young patients with colorectal cancer. $N$ Engl $J$ Med. 2000;342(2):69-77.

10. Phipps AI, Limburg PJ, Baron JA, et al. Association between molecular subtypes of colorectal cancer and patient survival. Gastroenterology. 2015;148(1):77-87.e2.

11. Malesci A, Laghi L, Bianchi P, et al. Reduced likelihood of metastases in patients with microsatellite-unstable colorectal cancer. Clin Cancer Res. 2007;13(13):3831-3839.

12. Ribic CM, Sargent DJ, Moore MJ, et al. Tumor microsatellite-instability status as a predictor of benefit from fluorouracil-based adjuvant chemotherapy for colon cancer. $N$ Engl J Med. 2003;349(3):247-257.

13. Sargent DJ, Marsoni S, Monges G, et al. Defective mismatch repair as a predictive marker for lack of efficacy of fluorouracil-based adjuvant therapy in colon cancer. J Clin Oncol. 2010;28(20):3219-3226.

14. Roth AD, Delorenzi M, Tejpar S, et al. Integrated analysis of molecular and clinical prognostic factors in stage II/III colon cancer. J Natl Cancer Inst. 2012;104(21):1635-1646.

15. Hutchins G, Southward K, Handley K, et al. Value of mismatch repair, KRAS, and BRAF mutations in predicting recurrence and benefits from chemotherapy in colorectal cancer. J Clin Oncol. 2011;29(10): 1261-1270.

16. Goldstein J, Tran B, Ensor J, et al. Multicenter retrospective analysis of metastatic colorectal cancer (CRC) with high-level microsatellite instability (MSI-H). Ann Oncol. 2014;25(5):1032-1038.

17. Brueckl WM, Moesch C, Brabletz T, et al. Relationship between microsatellite instability, response and survival in palliative patients with colorectal cancer undergoing first-line chemotherapy. Anticancer Res. 2003;23(2C):1773-1777.

18. Bertagnolli MM, Niedzwiecki D, Compton CC, et al. Microsatellite instability predicts improved response to adjuvant therapy with irinotecan, fluorouracil, and leucovorin in stage III colon cancer: cancer and leukemia group B protocol 89803. J Clin Oncol. 2009;27(11): 1814-1821.

19. de Vos tot Nederveen Cappel WH, Meulenbeld HJ, Kleibeuker JH, et al. Survival after adjuvant 5-FU treatment for stage III colon cancer in hereditary nonpolyposis colorectal cancer. Int J Cancer. 2004;109(3): $468-471$.

20. Kim ST, Lee J, Park SH, et al. Clinical impact of microsatellite instability in colon cancer following adjuvant FOLFOX therapy. Cancer Chemother Pharmacol. 2010;66(4):659-667.

21. Oh SY, Kim DY, Kim YB, Suh KW. Oncologic outcomes after adjuvant chemotherapy using FOLFOX in MSI-H sporadic stage III colon cancer. World J Surg. 2013;37(10):2497-2503.

22. Sinicrope FA, Mahoney MR, Smyrk TC, et al. Prognostic impact of deficient DNA mismatch repair in patients with stage III colon cancer from a randomized trial of FOLFOX-based adjuvant chemotherapy. J Clin Oncol. 2013;31(29):3664-3672. 
23. Venderbosch S, Nagtegaal ID, Maughan TS, et al. Mismatch repair status and BRAF mutation status in metastatic colorectal cancer patients: a pooled analysis of the CAIRO, CAIRO2, COIN, and FOCUS studies. Clin Cancer Res. 2014;20(20):5322-5330.

24. Le DT, Uram JN, Wang H, et al. PD-1 blockade in tumors with mismatchrepair deficiency. $N$ Engl J Med. 2015;372(26):2509-2520.

25. Fang WL, Chang SC, Lan YT, et al. Microsatellite instability is associated with a better prognosis for gastric cancer patients after curative surgery. World J Surg. 2012;36(9):2131-2138.

26. Oki E, Kakeji Y, Zhao Y, et al. Chemosensitivity and survival in gastric cancer patients with microsatellite instability. Ann Surg Oncol. 2009;16(9):2510-2515.

27. Rouprêt M, Fromont G, Azzouzi AR, et al. Microsatellite instability as predictor of survival in patients with invasive upper urinary tract transitional cell carcinoma. Urology. 2005;65(6):1233-1237.

28. Steinbakk A, Malpica A, Slewa A, et al. High frequency microsatellite instability has a prognostic value in endometrial endometrioid adenocarcinoma, but only in FIGO stage 1 cases. Anal Cell Pathol (Amst). 2010; 33(5):245-255.

29. Seppala TT, Bohm JP, Friman M, et al. Combination of microsatellite instability and BRAF mutation status for subtyping colorectal cancer. Br J Cancer. 2015;112(12):1966-1975.

30. Ward RL, Turner J, Williams R, et al. Routine testing for mismatch repair deficiency in sporadic colorectal cancer is justified. JPathol. 2005; 207(4):377-384.

31. Miyakura Y, Sugano K, Konishi F, et al. Extensive methylation of hMLH1 promoter region predominates in proximal colon cancer with microsatellite instability. Gastroenterology. 2001;121(6):1300-1309.

32. Laghi L, Bianchi P, Malesci A. Gender difference for promoter methylation pattern of hMLH1 and p16 in sporadic MSI colorectal cancer. Gastroenterology. 2003;124(4):1165-1166.

33. Sinicrope FA, Shi Q, Smyrk TC, et al. Molecular markers identify subtypes of stage III colon cancer associated with patient outcomes. Gastroenterology. 2015;148(1):88-99.

34. Clancy C, Burke JP, Kalady MF, Coffey JC. BRAF mutation is associated with distinct clinicopathological characteristics in colorectal cancer: a systematic review and meta-analysis. Colorectal Dis. 2013; 15(12):e711-e718.
35. Kim H, An JY, Noh SH, Shin SK, Lee YC, Kim H. High microsatellite instability predicts good prognosis in intestinal-type gastric cancers. J Gastroenterol Hepatol. 2011;26(3):585-592.

36. Hankey BF, Ries LA, Edwards BK. The surveillance, epidemiology, and end results program: a national resource. Cancer Epidemiol Biomarkers Prev. 1999;8(12):1117-1121.

37. NIH [webpage on the Internet]. Surveillance, Epidemiology, and End Results Program (SEER 18 2005-2011) Database. Available from: http://seer.cancer.gov/statfacts/html/colorect.html. Accessed February 8, 2017.

38. Golan T, Urban D, Berger R, Lawrence YR. Changing prognosis of metastatic colorectal adenocarcinoma: differential improvement by age and tumor location. Cancer. 2013;119(16):3084-3091.

39. Klingbiel D, Saridaki Z, Roth AD, Bosman F, Delorenzi M, Tejpar S. Prognosis of stage II and III colon carcinoma treated with adjuvant 5-FU or FOLFIRI in relation to microsatellite status, results of the PETACC-3 trial. Ann Oncol. 2015;26(1):126-132.

40. Des Guetz G, Schischmanoff O, Nicolas P, Perret GY, Morere JF, Uzzan B. Does microsatellite instability predict the efficacy of adjuvant chemotherapy in colorectal cancer? A systematic review with metaanalysis. Eur J Cancer. 2009;45(10):1890-1896.

41. Ooki A, Akagi K, Yatsuoka T, et al. Combined microsatellite instability and BRAF gene status as biomarkers for adjuvant chemotherapy in stage III colorectal cancer. J Surg Oncol. 2014;110(8):982-988.

42. von Knebel Doeberitz M, Kloor M. Towards a vaccine to prevent cancer in Lynch syndrome patients. Fam Cancer. 2013;12(2):307-312.

43. Ford JM. Is breast cancer a part of Lynch syndrome? Breast Cancer Res. 2012;14(4):110.

44. Grandval P, Barouk-Simonet E, Bronner M, et al. Is the controversy on breast cancer as part of the Lynch-related tumor spectrum still open? Fam Cancer. 2012;11(4):681-683.

45. Win AK, Lindor NM, Jenkins MA. Risk of breast cancer in Lynch syndrome: a systematic review. Breast Cancer Res. 2013;15(2):R27.

46. Harkness EF, Barrow E, Newton K, et al. Lynch syndrome caused by MLH1 mutations is associated with an increased risk of breast cancer: a cohort study. J Med Genet. 2015;52(8):553-556.
OncoTargets and Therapy

\section{Publish your work in this journal}

OncoTargets and Therapy is an international, peer-reviewed, open access journal focusing on the pathological basis of all cancers, potential targets for therapy and treatment protocols employed to improve the management of cancer patients. The journal also focuses on the impact of management programs and new therapeutic agents and protocols on

\section{Dovepress}

patient perspectives such as quality of life, adherence and satisfaction The manuscript management system is completely online and includes a very quick and fair peer-review system, which is all easy to use. Visit http://www.dovepress.com/testimonials.php to read real quotes from published authors. 